International Journal of Pure and Applied Mathematics

Volume 99 No. $3 \quad 2015,373-383$

ISSN: 1311-8080 (printed version); ISSN: 1314-3395 (on-line version)

url: http://www.ijpam.eu

doi: http://dx.doi.org/10.12732/ijpam.v99i3.12

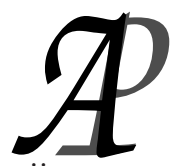

ijpam.eu

\title{
COMMON FIXED POINT THEOREMS IN \\ MODULAR METRIC SPACES
}

\author{
Hossein Rahimpoor $^{1 \S}$, Ali Ebadian ${ }^{2}$, Madjid Eshaghi Gordji ${ }^{3}$, Ali Zohri ${ }^{4}$ \\ ${ }^{1,2,4}$ Department of Mathematics \\ Payame Noor University \\ P.O. BOX 19395-3697, Tehran, IRAN \\ ${ }^{3}$ Department of Mathematics \\ Semnan University \\ P.O. BOX 35195-363, Semnan, IRAN \\ ${ }^{3}$ Center of Excellence in \\ onlinear Analysis and Applications (CENAA) \\ Semnan University \\ Semnan, IRAN \\ alizohri@gmail.com
}

AMS Subject Classification: 46A80, 47H10

Key Words: modular metric spaces, common fixed point, coincidence point

\section{Introduction}

The study of fixed and common fixed points of mappings satisfying a certain metrical contractive condition attracted many researchers, see for example ([13], [14], [6]). The concept of the commutativity has generalized in several ways. For this Sessa [15] has introduced the concept of weakly commuting. Then Jungck

Received: December 18, 2014

(c) 2015 Academic Publications, Ltd.

${ }^{\S}$ Correspondence author 
generalized this idea, first to compatible mappings [7] and then to weakly compatible mappings [8]. It can be easly verified that when the two mappings are commuting then they are compatible but not conversely. In 1998, Jungck and Rhoades [9] introduced the notion of weakly compatible and showed that compatible maps are weakly compatible but not conversely. In 2010, V. V. Chistyakov introduced the notation of modular metric spaces [2], he present some interesting applications for superposition operators by applying the theory of modular metric spaces [3]. Later, researchers have studied and extend fixed point problems in modular metric spaces, see for example ( [1], [4], [5], [10], [11], [12]). In this paper we prove some coincidence and common fixed point theorems for a contractive mapping in modular metric spaces. Our results generalize and extend results of Mongkolkeha et al. [10].

Definition 1. A metric modular on a non-empty set $X$ is a function $\omega:(0, \infty) \times X \times X \longrightarrow[0, \infty]$ that will be written as $\omega_{\lambda}(x, y)=\omega(\lambda, x, y)$ for all $x, y, z \in X$ and for all $\lambda>0$, satisfies the following three conditions:

(i) given $x, y \in X, \omega_{\lambda}(x, y)=0$ for all $\lambda>0$ iff $x=y$;

(ii) $\omega_{\lambda}(x, y)=\omega_{\lambda}(y, x)$, for all $\lambda>0$ and all $x, y \in X$;

(iii) $\omega_{\lambda+\mu}(x, y) \leq \omega_{\lambda}(x, z)+\omega_{\mu}(z, y)$ for all $\lambda, \mu>0$ and $x, y, z \in X$.

If instead of (i), we have only the condition

$\left(i_{1}\right) \omega_{\lambda}(x, x)=0$, then $\omega$ is said to be a (metric) pseudomodular on $X$ and if $\omega$ satisfies $\left(i_{1}\right)$ and

$\left(i_{2}\right)$ given $x, y \in X$, if there exists $\lambda>0$, possibly depending on $x$ and $y$ such that $\omega_{\lambda}(x, y)=0$ then $x=y$,

with this condition $\omega$ is called a strict modular on $X$.

If instead of $($ iii $)$ we replace the following condition:

$\left(i_{3}\right) \omega_{\lambda+\mu}(x, y) \leq \frac{\lambda}{\lambda+\mu} \omega_{\lambda}(x, z)+\frac{\mu}{\lambda+\mu} \omega_{\lambda}(z, y)$ for all $\lambda, \mu>0$ and $x, y, z \in X$; then $\omega$ is called a convex modular on $X$.

Remark 2. A modular $\omega$ on a set $X$, by $0<\lambda \longrightarrow \omega_{\lambda}(x, y) \in[0, \infty]$ for given $x, y \in X$, is nonincreasing on $(0, \infty)$. In fact, if $0<\lambda<\mu$, then by above definition

$$
\omega_{\mu}(x, y) \leq \omega_{\mu-\lambda}(x, x)+\omega_{\lambda}(x, y)=\omega_{\lambda}(x, y)
$$

for all $x, y \in X$.

If a metric modular $\omega$ on $X$ possesses a finite valued and $\omega$ is independent of $\lambda$ i.e; $\omega_{\lambda}(x, y)=\omega_{\mu}(x, y)$ for all $\lambda, \mu>0$, then $\omega_{\lambda}(x, y)$ is a metric on $X$. 
The binary relation $\stackrel{\omega}{\sim}$ on $X$ defined for each $x, y \in X$ by $x \stackrel{\omega}{\sim} y$ if and only if $\lim _{\lambda \rightarrow \infty} \omega_{\lambda}(x, y)=0$. That is an equivalence relation and denote by $X_{\omega}\left(x_{0}\right)$. The set $X_{\omega}$ is called a modular space. The modular space $X_{\omega}$ can be equipped with a metric $d_{\omega}$ given by

$$
d_{\omega}(x, y)=\inf \left\{\lambda>0: \omega_{\lambda}(x, y) \leq \lambda\right\}, \quad x, y \in X_{\omega}
$$

and the modular set $X_{\omega}$ is a metric space, see Theorem 2.6 of [2].

We also put

$X_{\omega}^{*} \equiv X_{\omega}^{*}\left(x_{0}\right)=\left\{x \in X: \exists \lambda=\lambda(x)>0\right.$ such that $\left.\omega_{\lambda}\left(x, x_{0}\right)<\infty\right\}$.

Theorem 3. Given a convex modular $\omega$ on a set $X$ and put

$$
d_{\omega}^{*}(x, y):=\inf \left\{\lambda>0: \omega_{\lambda}(x, y) \leq 1\right\}, \quad x, y \in X_{\omega}^{*} .
$$

Then $\left(X_{\omega}^{*}, d_{\omega}^{*}\right)$ is a metric space.

Proof. See Theorem 3.6 of [2].

Remark 4. $X_{\omega} \subset X_{\omega}^{*}$ and this inclusion is proper in general, and if $\omega$ is a convex modular on $X$, then two modular spaces defined as above are same, $X_{\omega}=X_{\omega}^{*}$. See [4].

Definition 5. [4] Given a modular $\omega$ on $X$. A sequence $\left\{x_{n}\right\} \equiv\left\{x_{n}\right\}_{n=1}^{\infty}$ in $X_{\omega}\left(X_{\omega}^{*}\right)$ is said to be modular convergent ( $\omega$-convergent) to an element $x \in X$ if there exists a number $\lambda>0$, possibly depending on $\left\{x_{n}\right\}$ and $x$, such that $\lim _{n \rightarrow \infty} \omega_{\lambda}\left(x_{n}, x\right)=0$. This will be written briefly as $x_{n} \stackrel{\omega}{\rightarrow} x$ as $n \rightarrow \infty$.

Definition 6. [4] Given a modular $\omega$ on $X$. A sequence $\left\{x_{n}\right\} \subset X_{\omega}$ in $X_{\omega}\left(X_{\omega}^{*}\right)$ is said to be modular Cauchy ( $\omega$-Cauchy) if there exists a number $\lambda=\lambda\left(\left\{x_{n}\right\}\right)>0$ such that $\lim _{m, n \rightarrow \infty} \omega_{\lambda}\left(x_{n}, x_{m}\right)=0$. i.e., for all $\varepsilon>$ 0 there exists $n_{0}(\varepsilon) \in \mathbb{N}$ such that for all $n, m \geq n_{0}(\varepsilon): \omega_{\lambda}\left(x_{n}, x_{m}\right) \leq \varepsilon$.

Definition 7. [4] Given a modular $\omega$ on $X$, the modular space $X_{\omega}$ is said to be modular complete ( $\omega$-complete) if each modular Cauchy sequence from $X_{\omega}$ is modular convergent. More precisely; if $\left\{x_{n}\right\} \subset X_{\omega}$ and there exists $\lambda=\lambda\left\{x_{n}\right\}>0$ such that

$$
\lim _{m, n \rightarrow \infty} \omega_{\lambda}\left(x_{n}, x_{m}\right)=0
$$


then there exists $x \in X_{\omega}$ such that $\lim _{n \rightarrow \infty} \omega_{\lambda}\left(x_{n}, x\right)=0$.

Remark 8. [2] Let $X_{\omega}$ be a modular metric space, then the metric convergent (with respect $d_{\omega}$ ) implies the modular convergent. i.e;

$$
\lim _{n \rightarrow \infty} d_{\omega}\left(x_{n}, x\right)=0 \text { if and only if } \lim _{n \rightarrow \infty} \omega_{\lambda}\left(x_{n}, x\right)=0 \text { for all } \lambda>0 .
$$

Definition 9. [4] A modular $\omega$ on $X$ is said to satisfy the $\Delta_{2}$-condition if for a sequence $\left\{x_{n}\right\} \subset X_{\omega}$ and $x \in X_{\omega}$, there exists a number $\lambda>0$, possibly depending on $\left\{x_{n}\right\}$ and $x$, such that $\lim _{n \rightarrow \infty} \omega_{\lambda}\left(x_{n}, x\right)=0$, then $\lim _{n \rightarrow \infty} \omega_{\frac{\lambda}{2}}\left(x_{n}, x\right)=0$. This implies that $\lim _{n \rightarrow \infty} \omega_{\lambda}\left(x_{n}, x\right)=0$ for all $\lambda>0$.

Note that in the all of this paper we suppose that $\omega$ is a modular on $X$ and satisfy in the $\Delta_{2}$-condition on $X$.

\section{Main result}

Let $X_{\omega}$ be a modular metric space and $S, T: X_{\omega} \longrightarrow X_{\omega}$. A point $x \in X_{\omega}$ is called a coincidence point of $S$ and $T$ if $T x=S x$. The mapping $S$ and $T$ are said to be weakly compatible if they commute at their coincidence point (i.e., $T S x=S T x$ whenever $T x=S x)$. Suppose $T\left(X_{\omega}\right) \subset S\left(X_{\omega}\right)$. Let $x_{0}$ be an arbitrary point in $X_{\omega}$. Since $T\left(X_{\omega}\right) \subseteq S\left(X_{\omega}\right)$ there exists a point $x_{1} \in X_{\omega}$ such that $T x_{0}=S x_{1}$. By repeating this process we construct the sequence $\left\{x_{n}\right\}$ in $X_{\omega}$ such that $S x_{n}=T x_{n-1}$ for all $n \geq 1$, we say that $\left\{T x_{n}\right\}$ is a $T-S$-sequence with initial point $x_{0}$.

Theorem 10. Let $X_{\omega}$ be a modular metric space and $S, T: X_{\omega} \longrightarrow X_{\omega}$ be two mapping such that $T\left(X_{\omega}\right) \subseteq S\left(X_{\omega}\right)$ and $S\left(X_{\omega}\right)$ be a $\omega$-complete subspace of $X_{\omega}$. Suppose there exists numbers $\alpha, \beta, \gamma \in[0,1)$ such that the following assertion for all $x, y \in X_{\omega}$ and $\lambda>0$ hold:

1. $(\alpha+2 \beta+2 \gamma)<1$ for all $0 \leq \alpha, \beta, \gamma<1$;

2.

$$
\begin{array}{r}
\omega_{\lambda}(T x, T y) \leq \alpha \omega_{\lambda}(S x, S y)+\beta\left[\omega_{\lambda}(S x, T x)+\omega_{\lambda}(S y, T y)\right] \\
+\gamma\left[\omega_{2 \lambda}(S x, T y)+\omega_{\lambda}(S y, T x)\right] ;
\end{array}
$$

3. $\omega_{\lambda}(S x, T y)<\infty$.

Then $T$ and $S$ have a coincidence point. 
Proof. Let $x_{0}$ be an arbitrary point in $X_{\omega}$. Since $T\left(X_{\omega}\right) \subseteq S\left(X_{\omega}\right)$ there exists a $T-S-$ sequence $\left\{T x_{n}\right\}$ in $X_{\omega}$ such that

$$
S x_{n}=T x_{n-1}
$$

for all $n \geq 1$. Now we take $x=x_{n}$ and $y=x_{n+1}$ in (2), we get

$$
\begin{aligned}
\omega_{\lambda}\left(T x_{n}, T x_{n+1}\right) & \leq \alpha \omega_{\lambda}\left(S x_{n}, S x_{n+1}\right) \\
& +\beta\left[\omega_{\lambda}\left(S x_{n}, S x_{n+1}\right)+\omega_{\lambda}\left(T x_{n}, T x_{n+1}\right)\right] \\
& +\gamma\left[\omega_{2 \lambda}\left(T x_{n-1}, T x_{n+1}\right)+\omega_{\lambda}\left(S x_{n+1}, S x_{n+1}\right)\right]
\end{aligned}
$$

for all $\lambda>0$. On the other hand

$$
\begin{aligned}
\omega_{2 \lambda}\left(T x_{n-1}, T_{n+1}\right) & \leq \omega_{\lambda}\left(T x_{n-1}, T x_{n}\right)+\omega_{\lambda}\left(T x_{n}, T x_{n+1}\right) \\
& =\omega_{\lambda}\left(S x_{n}, S x_{n+1}\right)+\omega_{\lambda}\left(T x_{n}, T x_{n+1}\right)
\end{aligned}
$$

so we obtain,

$$
\begin{aligned}
\omega_{\lambda}\left(T x_{n}, T x_{n+1}\right) & \leq \alpha \omega_{\lambda}\left(S x_{n}, S x_{n+1}\right) \\
& +\beta\left[\omega_{\lambda}\left(S x_{n}, S x_{n+1}\right)+\omega_{\lambda}\left(T x_{n}, T x_{n+1}\right)\right] \\
& +\gamma\left[\omega_{\lambda}\left(T x_{n}, T x_{n+1}\right)+\omega_{\lambda}\left(S x_{n}, S x_{n+1}\right)\right] .
\end{aligned}
$$

This implies that

$$
\omega_{\lambda}\left(T x_{n}, T x_{n+1}\right) \leq k \omega_{\lambda}\left(S x_{n}, S_{n+1}\right) \text { for all } n \in \mathbb{N}
$$

where $k=\frac{\alpha+\beta+\gamma}{1-\beta-\gamma}<1$. So by induction we get

$$
\omega_{\lambda}\left(T x_{n}, T x_{n+1}\right) \leq k^{n} \omega_{\lambda}\left(T x_{0}, T x_{1}\right) \text { for all } n \in \mathbb{N} \text {. }
$$

By 2, in a straightforward way, we imply that $\left\{T x_{n}\right\}$ is a $\omega$-Cauchy sequence. Since $S\left(X_{\omega}\right)$ is $\omega$-complete, there exists $u, v \in X_{\omega}$ such that $u=S(v)$ and $T x_{n} \stackrel{\omega}{\rightarrow} u$ as $n \rightarrow \infty$. Since $\omega$ satisfy in the $\Delta_{2}$-condition on $X$ we get $\lim _{n \rightarrow \infty} \omega_{\lambda}\left(T x_{n}, u\right)=0$ for all $\lambda>0$, therefore

$$
\lim _{n \rightarrow \infty} \omega_{\lambda}\left(T x_{n}, u\right)=\lim _{n \rightarrow \infty} \omega_{\lambda}\left(S x_{n}, u\right)=0 \text { for all } \lambda>0 .
$$

Now by taking $x=x_{n}$ and $y=v$ in (2), we obtain that

$$
\begin{aligned}
\omega_{\lambda}\left(T x_{n}, T v\right) & \leq \alpha \omega_{\lambda}\left(S x_{n}, S v\right)+\beta\left[\omega_{\lambda}\left(S x_{n}, T x_{n}\right)+\omega_{\lambda}(S v, T v)\right] \\
& +\gamma\left[\omega_{2 \lambda}\left(S x_{n}, T v\right)+\omega_{\lambda}\left(S v, T x_{n}\right)\right]
\end{aligned}
$$


by Remark 2 the function $\lambda \longmapsto \omega_{\lambda}(x, y)$ is non-increasing, so we have

$$
\begin{aligned}
\omega_{\lambda}\left(T x_{n}, T v\right) & \leq \alpha \omega_{\lambda}\left(S x_{n}, S v\right)+\beta\left[\omega_{\lambda}\left(S x_{n}, T x_{n}\right)+\omega_{\lambda}(S v, T v)\right] \\
& +\gamma\left[\omega_{\lambda}\left(S x_{n}, T x_{n}\right)+\omega_{\lambda}\left(T x_{n}, T v\right)+\omega_{\lambda}\left(S v, T x_{n}\right)\right]
\end{aligned}
$$

By using 3 and letting $n \rightarrow \infty$ in the above inequality, we get

$$
\begin{aligned}
\omega_{\lambda}(S v, T v) & \leq \alpha \omega_{\lambda}(S v, S v)+\beta\left[\omega_{\lambda}(S v, T v)+\omega_{\lambda}(S v, T v)\right] \\
& +\gamma\left[\omega_{\lambda}(S v, T v)+\omega_{\lambda}(S v, T v)+\omega_{\lambda}(S v, S v)\right]
\end{aligned}
$$

So $(1-2 \beta-2 \gamma) \omega_{\lambda}(S v, T v) \leq 0$, for all $\lambda>0$, and hence

$$
S v=T v=u \text {. }
$$

Thus we have proved that $S$ and $T$ have a coincidence point.

Theorem 11. In addition to the hypotheses of Theorem 10, suppose that $T$ and $S$ are weakly compatible, then $T$ and $S$ have a unique common fixed point. Further, for any $x_{0} \in X_{\omega}$, the $T-S$ - sequence $\left\{T x_{n}\right\}$ with initial point $x_{0}$ modular converges to the common fixed point.

Proof. Assume that $S, T$ are weakly compatible, then

$$
S u=S T v=T S v=T u,
$$

we will show that $T u=u=T v$.

Suppose $\omega_{\lambda}(T u, T v)>0$ for all $\lambda>0$, by taking $x=u$ and $y=v$ in (2) we get

$$
\begin{aligned}
\omega_{\lambda}(T u, T v) & \leq \alpha \omega_{\lambda}(S u, S v)+\beta\left[\omega_{\lambda}(S u, T u)+\omega_{\lambda}(S v, T v)\right] \\
& +\gamma\left[\omega_{2 \lambda}(S u, T v)+\omega_{\lambda}(S v, T u)\right]
\end{aligned}
$$

for all $u, v \in X_{\omega}$ and $\lambda>0$, i.e.,

$$
\begin{aligned}
\omega_{\lambda}(T u, T v) & \leq \alpha \omega_{\lambda}(T u, T v)+\beta\left[\omega_{\lambda}(T u, T u)+\omega_{\lambda}(T v, T v)\right] \\
& +\gamma\left[\omega_{2 \lambda}(T u, T v)+\omega_{\lambda}(T v, T u)\right]
\end{aligned}
$$

by Remark 2 we have

$$
\omega_{\lambda}(T u, T v) \leq \alpha \omega_{\lambda}(T u, T v)+\gamma\left[\omega_{\lambda}(T u, T v)+\omega_{\lambda}(T v, T u)\right]
$$

for all $\lambda>0$. This implies that $\omega_{\lambda}(T u, T v)(1-\alpha-2 \gamma) \leq 0$, which is a contradiction by assumption. 
Therefore $S u=T u=T v=u$, and hence $S, T$ have common fixed point. For the uniqueness of the common fixed point, suppose that $u$ and $z$ be two common fixed point, i.e.,

$$
T u=S u=u \quad \text { and } \quad T z=S z=z .
$$

By taking $x=u$ and $y=z$ in (2), we obtain that

$$
\begin{aligned}
\omega_{\lambda}(T u, T z) & \leq \alpha \omega_{\lambda}(S u, S z)+\beta\left[\omega_{\lambda}(S u, T u)+\omega_{\lambda}(S z, T z)\right] \\
& +\gamma\left[\omega_{2 \lambda}(S u, T z)+\omega_{\lambda}(S z, T u)\right]
\end{aligned}
$$

for all $\lambda>0$. So, $\omega_{\lambda}(T u, T z)(1-\alpha-2 \gamma) \leq 0$ for all $\lambda>0$, which is contradiction. Therefore $\omega_{\lambda}(u, z)=0$ for all $\lambda>0$ and so $u=z$. Clearly, for any $x_{0} \in X_{\omega}$, the $T-S$-sequence $\left\{T x_{n}\right\}$ with initial point $x_{0}$ converges to the unique common fixed point.

By setting $S=I_{X_{\omega}}$, we deduce the following result of fixed point for one self-mapping from Theorem 10.

Corollary 12. Let $X_{\omega}$ be a $\omega$-complete modular metric space and $T$ : $X_{\omega} \longrightarrow X_{\omega}$, such that for all $\lambda>0$ and $x, y \in X_{\omega}, \omega_{\lambda}(x, T y)<\infty$ and

$$
\begin{aligned}
\omega_{\lambda}(T x, T y) & \leq \alpha \omega_{\lambda}(x, y)+\beta\left[\omega_{\lambda}(x, T x)+\omega_{\lambda}(y, T y)\right] \\
& +\gamma\left[\omega_{2 \lambda}(x, T y)+\omega_{\lambda}(y, T x)\right]
\end{aligned}
$$

where $(\alpha+2 \beta+2 \gamma)<1$ and $0 \leq \alpha, \beta, \gamma<1$. Then $T$ has a unique fixed point. Further, for any $x_{0} \in X_{\omega}$, the Picard sequence $\left\{T x_{n}\right\}$ with initial point $x_{0}$ modular converges to the fixed point.

Corollary 13. Let $X_{\omega}$ be a $\omega$-complete modular metric space and $T$ : $X_{\omega} \longrightarrow X_{\omega}$, such that for all $\lambda>0$ and $x, y \in X_{\omega}, \omega_{\lambda}(x, T y)<\infty$ and

$$
\omega_{\lambda}(T x, T y) \leq \alpha \omega_{\lambda}(x, y)
$$

where $0 \leq \alpha<1$. Then $T$ has a unique fixed point.

Corollary 14. Let $X_{\omega}$ be a $\omega$-complete modular metric space and $T$ : $X_{\omega} \longrightarrow X_{\omega}$, such that for all $\lambda>0$ and $x, y \in X_{\omega}, \omega_{\lambda}(x, T y)<\infty$ and

$$
\omega_{\lambda}(T x, T y) \leq \beta\left[\omega_{\lambda}(x, T x)+\omega_{\lambda}(y, T y)\right]
$$

where $\beta \in\left[0, \frac{1}{2}\right)$. Then $T$ has a unique fixed point. 
Recently, Mongkolkeha et al. [10] has introduced some notions and established some fixed point results in modular metric spaces. The Corollaries of 13 and 14 are results of [10].

Theorem 15. Let $X_{\omega}$ be a modular metric space and $S, T: X_{\omega} \longrightarrow X_{\omega}$ be two mapping such that $T\left(X_{\omega}\right) \subseteq S\left(X_{\omega}\right)$ and $S\left(X_{\omega}\right)$ be a $\omega$-complete subspace of $X_{\omega}$. Suppose there exists mappings $\alpha, \beta, \gamma, \mu: X_{\omega} \longrightarrow[0,1)$ such that the following assertion for all $x, y \in X_{\omega}$ and $\lambda>0$ hold:

1. $\alpha(T x) \leq \alpha(S x), \beta(T x) \leq \beta(S x), \gamma(T x) \leq \gamma(S x), \mu(T x) \leq \mu(S x)$;

2. $(\alpha+2 \beta+\gamma+\mu)<1$ for all $0 \leq \alpha, \beta, \gamma, \mu<1$;

3. $\omega_{\lambda}(T x, T y) \leq \alpha(S x) \omega_{\lambda}(S x, S y)+\beta(S x) \omega_{2 \lambda}(S x, T y)+\gamma(S x) \omega_{\lambda}(S x, T x)+$ $\mu(S x) \omega_{\lambda}(S y, T y)$

4. $\omega_{\lambda}(S x, T y)<\infty$.

Then $T$ and $S$ have a unique common fixed point.

Proof. Let $x_{0}$ be an arbitrary point in $X_{\omega}$. Since $T\left(X_{\omega}\right) \subseteq S\left(X_{\omega}\right)$ there exists a $T-S-$ sequence $\left\{T x_{n}\right\}$ in $X_{\omega}$ such that

$$
S x_{n}=T x_{n-1}
$$

for all $n \geq 1$. From (1),(3) and 4, we have

$$
\begin{aligned}
\omega_{\lambda}\left(S x_{n}, S x_{n+1}\right) & =\omega_{\lambda}\left(T x_{n-1}, T x_{n}\right) \\
\leq & \alpha\left(S x_{n-1}\right) \omega_{\lambda}\left(S x_{n-1}, S x_{n}\right)+\beta\left(S x_{n-1}\right) \omega_{2 \lambda}\left(S x_{n-1}, T x_{n}\right) \\
& +\gamma\left(S x_{n-1}\right) \omega_{\lambda}\left(S x_{n-1}, T x_{n-1}\right)+\mu\left(S x_{n-1}\right) \omega_{\lambda}\left(S x_{n}, T x_{n}\right) \\
& =\alpha\left(T x_{n-2}\right) \omega_{\lambda}\left(S x_{n-1}, S x_{n}\right)+\beta\left(T x_{n-2}\right) \omega_{2 \lambda}\left(S x_{n-1}, S x_{n+1}\right) \\
& +\gamma\left(T x_{n-2}\right) \omega_{\lambda}\left(S x_{n-1}, S x_{n}\right)+\mu\left(T x_{n-2}\right) \omega_{\lambda}\left(S x_{n}, S x_{n+1}\right) \\
\leq & \alpha\left(S x_{n-2}\right) \omega_{\lambda}\left(S x_{n-1}, S x_{n}\right)+\beta\left(S x_{n-2}\right) \omega_{2 \lambda}\left(S x_{n-1}, S x_{n+1}\right) \\
& +\gamma\left(S x_{n-2}\right) \omega_{\lambda}\left(S x_{n-1}, S x_{n}\right)+\mu\left(S x_{n-2}\right) \omega_{\lambda}\left(S x_{n}, S x_{n+1}\right) \\
& \cdot \gamma\left(S x_{0}\right) \omega_{\lambda}\left(S x_{n-1}, S x_{n}\right)+\beta\left(S x_{0}\right) \omega_{2 \lambda}\left(S x_{n-1}, S x_{n+1}\right) \\
& \cdot \gamma\left(S x_{0}\right) \omega_{\lambda}\left(S x_{n-1}, S x_{n}\right)+\mu\left(S x_{0}\right) \omega_{\lambda}\left(S x_{n}, S x_{n+1}\right) \\
\leq & \alpha\left(S x_{0}\right) \omega_{\lambda}\left(S x_{n-1}, S x_{n}\right)+\beta\left(S x_{0}\right)\left[\omega_{\lambda}\left(S x_{n-1}, S x_{n}\right)\right. \\
& \left.+\omega_{\lambda}\left(S x_{n}, S x_{n+1}\right)\right] \\
& +\gamma\left(S x_{0}\right) \omega_{\lambda}\left(S x_{n-1}, S x_{n}\right)+\mu\left(S x_{0}\right) \omega_{\lambda}\left(S x_{n}, S x_{n+1}\right) . \\
&
\end{aligned}
$$


This implies that

$$
\omega_{\lambda}\left(S x_{n}, S x_{n+1}\right) \leq\left(\frac{\alpha\left(S x_{0}\right)+\beta\left(S x_{0}\right)+\gamma\left(S x_{0}\right)}{1-\beta\left(S x_{0}\right)-\mu\left(S x_{0}\right)}\right) \omega_{\lambda}\left(S x_{n-1}, S x_{n}\right)
$$

for all $n \geq 1$. Now, we let

$$
k=\frac{\alpha\left(S x_{0}\right)+\beta\left(S x_{0}\right)+\gamma\left(S x_{0}\right)}{1-\beta\left(S x_{0}\right)-\mu\left(S x_{0}\right)}<1 .
$$

By repeating 5, we get

$$
\omega_{\lambda}\left(S x_{n}, S x_{n+1}\right) \leq k^{n} \omega_{\lambda}\left(S x_{1}, S x_{0}\right) .
$$

Now for $m>n \geq 1$, it follows from 6 that

$$
\begin{aligned}
\omega_{\lambda}\left(S x_{n}, S x_{m}\right) & \leq \omega_{\frac{\lambda}{m-n}}\left(S x_{n}, S x_{n+1}\right)+\omega_{\frac{\lambda}{m-n}}\left(S x_{n+1}, S x_{n+2}\right) \\
& +\ldots+\omega_{\frac{\lambda}{m-n}}\left(S x_{m-1}, S x_{m}\right) \\
& \leq\left(k^{n}+k^{n+1}+\ldots+k^{m-1}\right) \omega_{\frac{\lambda}{m-n}}\left(S x_{0}, S x_{1}\right) \\
& =\frac{k^{n}-k^{m}}{1-k} \omega_{\frac{\lambda}{m-n}}\left(S x_{0}, S x_{1}\right) .
\end{aligned}
$$

Since $0 \leq k<1$, we conclude that $\left\{S x_{n}\right\}$ is a $\omega$-Cauchy sequence in $S\left(X_{\omega}\right)$, on the other hand by hypothesis $S\left(X_{\omega}\right)$ is a $\omega$-complete subspace of $X_{\omega}$, therefore there exists a point $u \in S\left(X_{\omega}\right)$ such that $S x_{n} \stackrel{\omega}{\rightarrow} u$ as $n \rightarrow \infty$. By hypothesis $\omega$ satisfies in $\Delta_{2}$-condition on $X_{\omega}$, so $\lim _{n \rightarrow \infty} \omega_{\lambda}\left(S x_{n}, u\right)=0$ for all $\lambda>0$.

Now we claim that $u$ is common fixed point. Suppose that $T u \neq u$ or $S u \neq u$. Then we have

$$
\begin{aligned}
0 & <\inf \left\{\omega_{\lambda}(T x, u)+\omega_{\lambda}(S x, u)+\omega_{\lambda}(T x, S x): x \in X_{\omega}\right\} \\
& \leq \inf \left\{\omega_{\lambda}\left(T x_{n}, u\right)+\omega_{\lambda}\left(S x_{n}, u\right)+\omega_{\lambda}\left(T x_{n}, S x_{n}\right): n \geq 1\right\} \\
& =\inf \left\{\omega_{\lambda}\left(S x_{n+1}, u\right)+\omega_{\lambda}\left(S x_{n}, u\right)+\omega_{\lambda}\left(S x_{n}, S x_{n+1}\right)\right\} \\
& \leq \inf \left\{\omega_{\lambda}\left(S x_{n+1}, u\right)+\omega_{\lambda}\left(S x_{n}, u\right)+\omega_{\frac{\lambda}{2}}\left(S x_{n}, u\right)+\omega_{\frac{\lambda}{2}}\left(u, S x_{n+1}\right)\right\} \rightarrow 0
\end{aligned}
$$

as $n \rightarrow \infty$ for all $\lambda>0$, which is contradiction. Therefore, this implies that $u=S u=T u$.

For the uniqueness of the common fixed point, suppose $T u=S u=u$ and $T z=S z=z$ be two common fixed points, then by taking $x=u$ and $y=z$ in (3), we obtain that

$$
\omega_{\lambda}(u, z) \leq \alpha(u) \omega_{\lambda}(u, z)+\beta(u) \omega_{2 \lambda}(u, z)+\gamma(u) \omega_{\lambda}(u, u)+\mu(u) \omega_{\lambda}(z, z)
$$




$$
\leq \alpha(u) \omega_{\lambda}(u, z)+\beta(u) \omega_{\lambda}(u, z) .
$$

This implies that $(1-\alpha-\beta)(u)\left(\omega_{\lambda}(u, z)\right) \leq 0$, which is a contradiction by assumption.

By taking the mapping $S$ in Theorem 15 as $I_{X_{\omega}}$ where $I_{X_{\omega}}$ is an identity mapping on $X_{\omega}$, we have the following corollary.

Corollary 16. Let $X_{\omega}$ be a $\omega$-complete modular metric space and $T$ : $X_{\omega} \longrightarrow X_{\omega}$. Suppose there exists mappings $\alpha, \beta, \gamma, \mu: X_{\omega} \longrightarrow[0,1)$ such that the following assertion for all $x, y \in X_{\omega}$ and $\lambda>0$ hold:

1. $\alpha(T x) \leq \alpha(x), \beta(T x) \leq \beta(x), \gamma(T x) \leq \gamma(x), \mu(T x) \leq \mu(x)$;

2. $(\alpha+2 \beta+\gamma+\mu)<1$ for all $0 \leq \alpha, \beta, \gamma, \mu<1$;

3. $\omega_{\lambda}(T x, T y) \leq \alpha(x) \omega_{\lambda}(x, y)+\beta(x) \omega_{2 \lambda}(x, T y)+\gamma(x) \omega_{\lambda}(x, T x)+\mu(x) \omega_{\lambda}(y, T y)$;

4. $\omega_{\lambda}(x, T y)<\infty$.

Then $T$ has a unique fixed point.

\section{References}

[1] P. Chaipunya, C. Mongkolkeha, W. Sintunavarat, P. Kumam, FixedPoint Theorems for Multivalued Mappings in Modular Metric Spaces, Abstract and Applied Analysis 2012 (2012), Article ID 503504, 14 pages doi:10.1155/2012/503504

[2] V. Chistyakov, Modular metric spaces, I: Basic concepts, Nonlinear Analysis 72(2010)1-14, doi:10.1016/j.na.2009.04.057.

[3] V. Chistyakov, Modular metric spaces, II:Application to superposition operators, Nonlinear Analysis 72(2010)15-30, doi:10.1016/j.na.2009.04.018.

[4] V. Chistyakov, A fixed point theorem for contractions in modular metric spaces, Perprint submited to arxiv (2011).

[5] Y. Cho, R. Saadati, G. Sadeghi, Quasi-contraction mapping in modular metric spaces, Journal of Applied Mathematics 2012 (2012), Article ID 907951, 5 pages doi:10.1155/2012/907951.

[6] B. Fisher, Common fixed point for four mappings, Bull. Inst. of Math. Academia. Sinicia,11(1983), 103-113.

[7] G. Jungck, Compatible mappings and common fixed points, Int. J. Math. Math. Sci. 9(1986), 771-779, doi:10.1135/s0161171286000935. 
[8] G. Jungck, Common fixed points for noncontinuous nonself maps on nonmetric spaces, Far East J. Math. Sci. 4(1996), 19-215.

[9] G. Jungck, B. E. Rhoades, Fixed point for set valued functions without continuity, Indian J. Pure Appl. Math,29(3)(1998), 227-238.

[10] C. Mongkolkeha, W. Sintunavarat, P. Kumam, Fixed point theorems for contraction mappings in modular metric spaces, Fixed Point Theory and Applications, 2011, 2011:93 doi:10.1186/1687-1812-2011-93

[11] H. Rahimpoor, A. Ebadian, M. Eshaghi Gordji, A. Zohri, fixed point theory for generalized quasi-contraction maps in modular metric spaces, Journal of Mathematics and Computer Science. 10(2014)54-60

[12] H. Rahimpoor, A. Ebadian, M. Eshaghi Gordji, A. Zohri, Some fixed point theorems on modular metric spaces, Acta Universitatis Apulensis 37(2014)161-170.

[13] IA. Rus, Generalized contractions and applications, Cluj University Press, Cluj-Napoca(2001)

[14] IA. Rus, A. Petruşel, G. Petruşel, Fixed Point Theory, Cluj University Press, Cluj-Napoca(2008)

[15] S. Sessa, On a weak commutativity condition of mappings in fixed point considerations, Publ. Inst. Math. 32(1982), 149-153. 
